\title{
Peripapillary pachychoroid syndrome
}

Phasukkijwatana, Nopasak ; Freund, K Bailey ; Dolz-Marco, Rosa ; Al-Sheikh, Mayss ; Keane, Pearse A ; Egan, Catherine A ; Randhawa, Sandeep ; Stewart, Jay M ; Liu, Qingyun ; Hunyor, Alex P ; Kreiger, Allan ; Nagiel, Aaron ; Lalane, Robert ; Rahimi, Mansour ; Lee, Won Ki ; Jampol, Lee Merrill ; Sarraf, David

DOI: https://doi.org/10.1097/IAE.0000000000001907

Posted at the Zurich Open Repository and Archive, University of Zurich ZORA URL: https://doi.org/10.5167/uzh-142176

Journal Article

Published Version

Originally published at:

Phasukkijwatana, Nopasak; Freund, K Bailey; Dolz-Marco, Rosa; Al-Sheikh, Mayss; Keane, Pearse A; Egan, Catherine A; Randhawa, Sandeep; Stewart, Jay M; Liu, Qingyun; Hunyor, Alex P; Kreiger, Allan; Nagiel, Aaron; Lalane, Robert; Rahimi, Mansour; Lee, Won Ki; Jampol, Lee Merrill; Sarraf, David (2018). Peripapillary pachychoroid syndrome. Retina, 38(9):1652-1667.

DOI: https://doi.org/10.1097/IAE.0000000000001907 


\title{
PERIPAPILLARY PACHYCHOROID SYNDROME
}

\author{
NOPASAK PHASUKKIJWATANA, PHD, MD, $* \dagger$ K. BAILEY FREUND, MD, $\$ \S$ \\ ROSA DOLZ-MARCO, MD, PHD, $\$ \S$ MAYSS AL-SHEIKH, MD, $\mathbb{T}^{* *}$ PEARSE A. KEANE, MD, $\dagger \dagger$ \\ CATHERINE A. EGAN, MD, $\dagger$ SANDEEP RANDHAWA, MD, $\$$ \$ JAY M. STEWART, MD, III \\ QINGYUN LIU, MD,III*** ALEX P. HUNYOR, MD, $+\dagger+\uparrow+*$ ALLAN KREIGER, MD,*
}

AARON NAGIEL, MD, PHD,* ROBERT LALANE, MD, ${ }^{*}$ MANSOUR RAHIMI, MD,* WON KI LEE, MD, $\S \S$

LEE MERRILL JAMPOL, MD, গIIII DAVID SARRAF, MD*****

\begin{abstract}
Purpose: To describe the features of peripapillary pachychoroid syndrome (PPS), a novel pachychoroid disease spectrum (PDS) entity.

Methods: Medical records of 31 eyes (16 patients) with choroidal thickening associated with intraretinal and/or subretinal fluid in the nasal macula extending from the disk were reviewed (patients with PPS). Choroidal thickness was compared with 2 age-matched cohorts: typical PDS (17 eyes with central serous chorioretinopathy or pachychoroid neovasculopathy) and 19 normal eyes.
\end{abstract}

Results: The patients with PPS were $81 \%$ men aged $71 \pm 7$ years. Peripapillary pachychoroid syndrome eyes displayed thicker nasal versus temporal macular choroids, unlike PDS eyes with thicker temporal macular choroids $(P<0.0001)$. Peripapillary intraretinal and/or subretinal fluid was often overlying dilated Haller layer vessels (pachyvessels). Fundus autofluorescence and fluorescein angiography illustrated peripapillary pigmentary mottling without focal leakage. Most PPS eyes (70\%) exhibited other PDS findings including serous pigment epithelial detachment or gravitational tracks. Indocyanine green angiography illustrated dilated peripapillary pachyvessels and choroidal hyperpermeability. The disk was usually crowded, with edema noted in 4/31 (13\%) eyes and mild late fluorescein disk leakage identified in half of the cases. Choroidal folds (77\%), short axial lengths (39\% less than $23 \mathrm{~mm}$ ), and hyperopia (86\%) were common.

Conclusion: Peripapillary pachychoroid syndrome is a distinct PDS variant, in which peripapillary choroidal thickening is associated with nasal macular intraretinal and/or subretinal fluid and occasional disk edema. Recognition of PPS is important to distinguish it from disorders with overlapping features such as posterior uveitis and neuro-ophthalmologic conditions.

RETINA 0:1-16, 2017

$\mathrm{T}$ he pachychoroid disease spectrum (PDS) refers to a group of retinochoroidal disorders that share distinctive choroidal findings identified with multimodal retinal imaging. These choroidal features include focal or diffuse choroidal thickening associated with reduced fundus tessellation, dilated Haller layer vessels (termed "pachyvessels") with thinning of the overlying inner choroid, and choroidal hyperpermeability demonstrated with indocyanine green angiography (ICGA). The PDS includes pachychoroid pigment epitheliopathy, ${ }^{1}$ focal choroidal excavation, ${ }^{2}$ central serous chorioretinopathy (CSC) ${ }^{3}$ pachychoroid neovasculopathy, ${ }^{4}$ and polypoidal choroidal vasculopathy. ${ }^{5}$

We have identified a novel PDS variant, in which pachychoroid features surround the optic nerve and are associated with intraretinal and/or subretinal fluid and optic nerve head edema in some eyes. Associated findings including serous pigment epithelial detachment, choroidal hyperpermeability, and pachyvessels suggest that this is a pachychoroid-driven entity, rather than a variant of uveal effusion syndrome (UES) as was suggested in a single-case report. ${ }^{6}$ As such, we propose the term "peripapillary pachychoroid syndrome" (PPS). The aim of this investigation is to report the features of this syndrome and to compare this disorder with both normal eyes and two typical PDS entities, CSC, and pachychoroid neovasculopathy.

\section{Methods}

\section{Subjects}

This retrospective, multicenter, observational case series was approved by the University of California Los 
Angeles Institutional Review Board. The study adhered to the tenets of the Declaration of Helsinki and was conducted in accordance with the Health Insurance Portability and Accountability Act (HIPAA) regulations.

Patients with peripapillary choroidal thickening and intraretinal and/or subretinal fluid in the nasal macular region extending from the temporal margin of the optic disk as noted with optical coherence tomography (OCT) were identified and comprised Group 1. Peripapillary choroidal thickening was defined by the presence of 1) a thicker choroid than would be expected for age and/or 2) choroidal thickening associated with Haller vessel dilation and overlying inner choroidal thinning. ${ }^{1,3,4} \mathrm{~Pa}-$ tients were excluded from this group if they demonstrated other causes of macular edema, unrelated to a PDS entity,

From the *Retinal Disorders and Ophthalmic Genetics Division, Stein Eye Institute, David Geffen School of Medicine at UCLA, Los Angeles, California; †Department of Ophthalmology, Faculty of Medicine Siriraj Hospital, Mahidol University, Bangkok, Thailand; \$Vitreous Retina Macula Consultants of New York, New York, New York; §Department of Ophthalmology, New York University School of Medicine, New York, New York; qDepartment of Ophthalmology, Doheny Eye Institute, David Geffen School of Medicine at UCLA, Los Angeles, California; **Department of Ophthalmology, University Hospital Zurich, University of Zurich, Zurich, Switzerland; † $†$ Medical Retina Service, Moorfields Eye Hospital NHS Foundation Trust, London, United Kingdom; \$+Associated Retinal Consultant, PC, Royal Oak, Michigan; §§Ophthalmology, Oakland University William Beaumont School of Medicine, Royal Oak, Michigan; TqIDepartment of Ophthalmology, UCSF, San Francisco, California; ***Department of Ophthalmology, Tongliao City Hospital, Tongliao, China; $\dagger+$ Retina Associates, Chatswood, Australia; \$\$Macular Research Unit, Save Sight Institute, University of Sydney, Sydney, Australia; $\S \S \S$ Ophthalmology, Seoul St. Mary’s Hospital, Catholic University of Korea, Seoul, Korea; Tषा Feinberg School of Medicine, Chicago, Illinois; and ****Greater Los Angeles, VA Healthcare Center, Los Angeles, California.

Supported by The Macula Foundation, Inc, New York, NY.

K. B. Freund is a consultant for Optovue (Fremont, California), Optos (Dunfermline, Scotland), Heidelberg Engineering (Heidelberg, Germany), Genentech (South San Francisco, California), and GrayBug Vision (Redwood City, California) and receives research support from Genentech/Roche (Basel, Switzerland). P. A. Keane is an advisory board member for Heidelberg, Allergan (Dublin, Republic of Ireland), Bayer Healthcare (Leverkusen, Germany), Topcon (Tokyo, Japan), Haag-Streit (Köniz, Switzerland), and Novartis (Basel, Switzerland), a consultant for Google DeepMind (London, UK) and Optos, and receives Clinician Scientist award (CS-2014-14-023) from the National Institute for Health Research. The views expressed in this publication are those of the author(s) and not necessarily those of the NHS, the National Institute for Health Research or the Department of Health. A. P. Hunyor is an advisory board member for Bayer Healthcare, Novartis and Alcon (Fort Worth, Texas). A. Nagiel is a consultant for Allergan. W. K. Lee is a consultant for Bayer Healthcare, Novartis, and Santen Pharmaceutical (Osaka, Japan) and receives research support from Allergan. D. Sarraf is a consultant for Amgen, Bayer Healthcare, Genentech, Novartis, and Optovue and receives research or financial support from Allergan, Genentech, Heidelberg, Optovue, and Regeneron (Tarrytown, New York). The remaining authors have no conflicting interests to disclose.

Reprint requests: David Sarraf, MD, Retinal Disorders and Ophthalmic Genetics Division, Stein Eye Institute, David Geffen School of Medicine at UCLA, Los Angeles, CA 90095; e-mail: dsarraf@ucla.edu including any evidence of ocular inflammation, macular edema due to diabetic macular edema or retinal vascular occlusion, radiation retinopathy, significant epiretinal membrane formation, any history of intraocular surgery within the past 6 months before presentation, or the use of medications known to be associated with cystoid macular edema. Eyes with clinical or imaging evidence of choroidal neovascularization were excluded from this cohort. Patients with cavitary optic disk anomalies (e.g., optic disk pit, optic disk coloboma), optic atrophy, glaucomatous optic neuropathy, or ocular tumors were also excluded.

Medical records and multimodal imaging findings were comprehensively reviewed. The data collected included age, sex, medical history, Snellen visual acuity, axial length and refraction, and the findings of complete ocular examination. Multimodal imaging included color fundus photography (Carl Zeiss Meditec, Dublin, CA; Topcon Medical Systems, Oakland, NJ; Optos, Dunfermline, Scotland), spectral domain OCT (SPECTRALIS; Heidelberg Engineering, Heidelberg, Germany), enhanced depth imaging OCT (EDI-OCT) (SPECTRALIS, Heidelberg Engineering), swept-source OCT (DRI OCT Triton, Topcon Medical Systems), fluorescein angiography (FA) (Carl Zeiss Meditec; SPECTRALIS, Heidelberg Engineering; Topcon Medical Systems; Optos), ICGA (SPECTRALIS; Heidelberg Engineering), and fundus autofluorescence (FAF) (SPECTRALIS, Heidelberg Engineering; Optos).

Eleven consecutive patients (17 eyes) older than or equal to 50 years with typical findings of CSC or pachychoroid neovasculopathy (i.e., patients with typical findings of PDS) were recruited from the practice of D.S. as a comparative control group and comprised Group 2. Central serous chorioretinopathy was defined by the presence of choroidal thickening within the macula associated with subretinal fluid and corresponding leakage on FA or macular retinal pigment epithelium (RPE) abnormalities with granular autofluorescence and gravitational tracks. Patients with CSC or pachychoroid features associated with shallow irregular RPE detachment harboring Type 1 neovascularization (pachychoroid neovasculopathy) were also included in Group 2. In addition, 19 age-matched normal eyes from 12 patients with no ocular disease other than early cataract, or a history of cataract surgery were included as normal control eyes (Group 3).

\section{Image Analysis}

Choroidal thickness measurement. Choroidal thickness measurements were performed using EDI-OCT images, and the caliper tool provided with the review software (Heidelberg Eye Explorer, v1.9.10.0 software; Heidelberg Engineering). Choroidal thickness 
was defined as the perpendicular distance between Bruch membrane and the choroidal scleral junction. The measurement was performed on a horizontal section passing through the central fovea at the following positions: 1) the center of the fovea (subfoveal), 2) $1,500 \mu \mathrm{m}$ nasal to the foveal center (N1.5), 3) 3,000 $\mu \mathrm{m}$ nasal to the foveal center (N3.0), 4) 1,500 $\mu \mathrm{m}$ temporal to the foveal center (T1.5), 5) 3,000 $\mu \mathrm{m}$ temporal to the foveal center (T3.0), and 6) $250 \mu \mathrm{m}$ temporal to Bruch membrane origin at the temporal disk margin (BMO250) (Figure 1A). Images were set to an aspect ratio of $1: 1 \mu \mathrm{m}$ before each measurement. The hyporeflective band corresponding to the suprachoroidal space when present was not included in the choroidal thickness measurement. ${ }^{7}$ If the cho- roidal scleral junction was not clearly identified, brightness and contrast of the image was adjusted, using the built-in adjustment tool, to best visualize this junction, and the outer choroidal border was identified by the line connecting the outer margin of the large choroidal vessel layer. When available, adjacent EDIOCT scans were reviewed to confirm the accuracy of identifying the choroidal scleral junction. In one patient, choroidal thickness was measured on sweptsource OCT images using Image J 1.51a software ${ }^{8}$ with appropriate scales.

Two independent trained graders (N.P. and M.A.) performed all the measurements. Large disagreements $(>100 \mu \mathrm{m})$ over readings were resolved by open adjudication between the readers ( 2 instances).

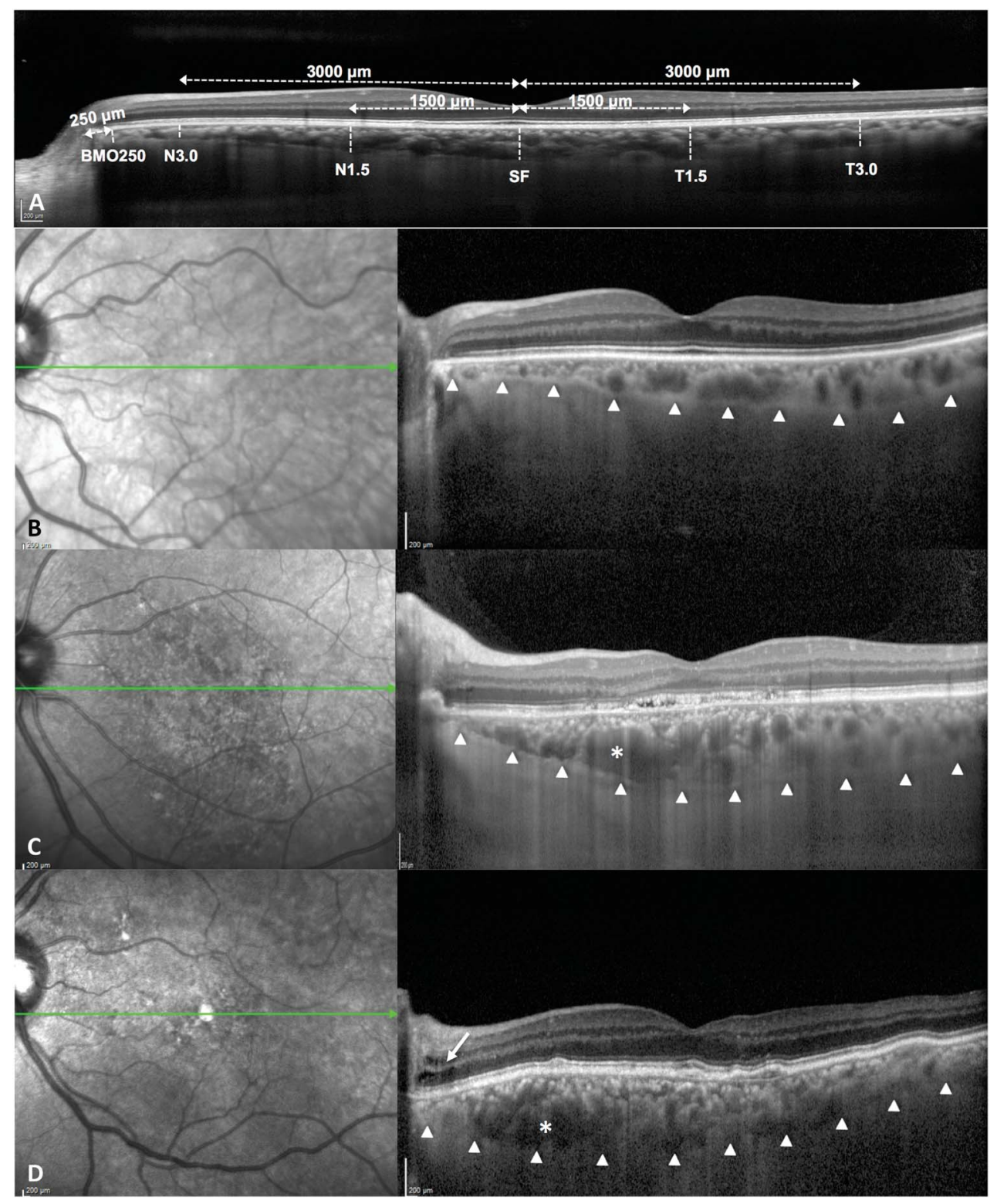

Fig. 1. Enhanced depth imaging OCT of the macula and choroid. A. Positions of choroidal thickness measurement used in this study. BMO250, $250 \mu \mathrm{m}$ temporal to Bruch membrane origin at the temporal disk margin; $\mathrm{N} 1.5,1,500 \mu \mathrm{m}$ nasal to the foveal center; N3.0, 3,000 $\mu \mathrm{m}$ nasal to the foveal center; SF, subfoveal; T1.5, 1,500 $\mu \mathrm{m}$ temporal to the foveal center; and T3.0, 3,000 $\mu \mathrm{m}$ temporal to the foveal center. B. Normal left eye of a 71-year-old woman. $\mathbf{C}$. Left eye of a 69-year-old man with typical chronic CSC. D. Left eye of a 75-year-old man with PPS. Note the peripapillary intraretinal cysts (arrow) and disproportionate thickening of the nasal choroid associated with choroidal folds in (D) versus the tapering nasal choroid in (B and C). Furthermore, there are more dilated large choroidal vessels (pachyvessels) on the nasal side compared with the temporal side in (D). White asterisks denote pachyvessels with associated thinning of the overlying inner choroid. Choroidal thickness is outlined with arrowheads. 

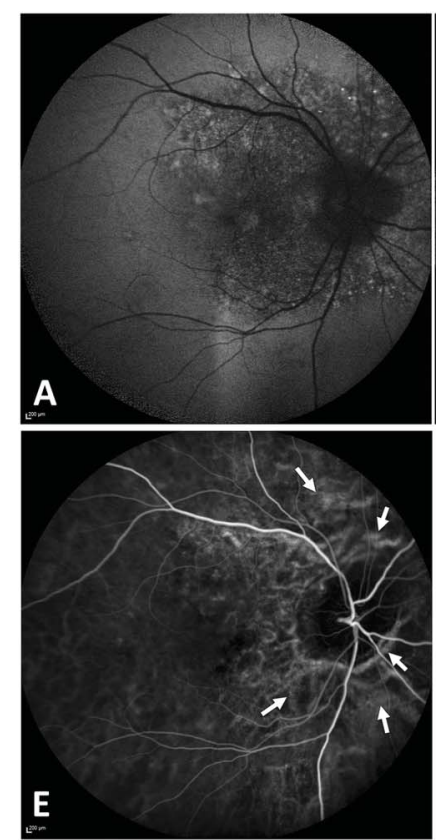

B
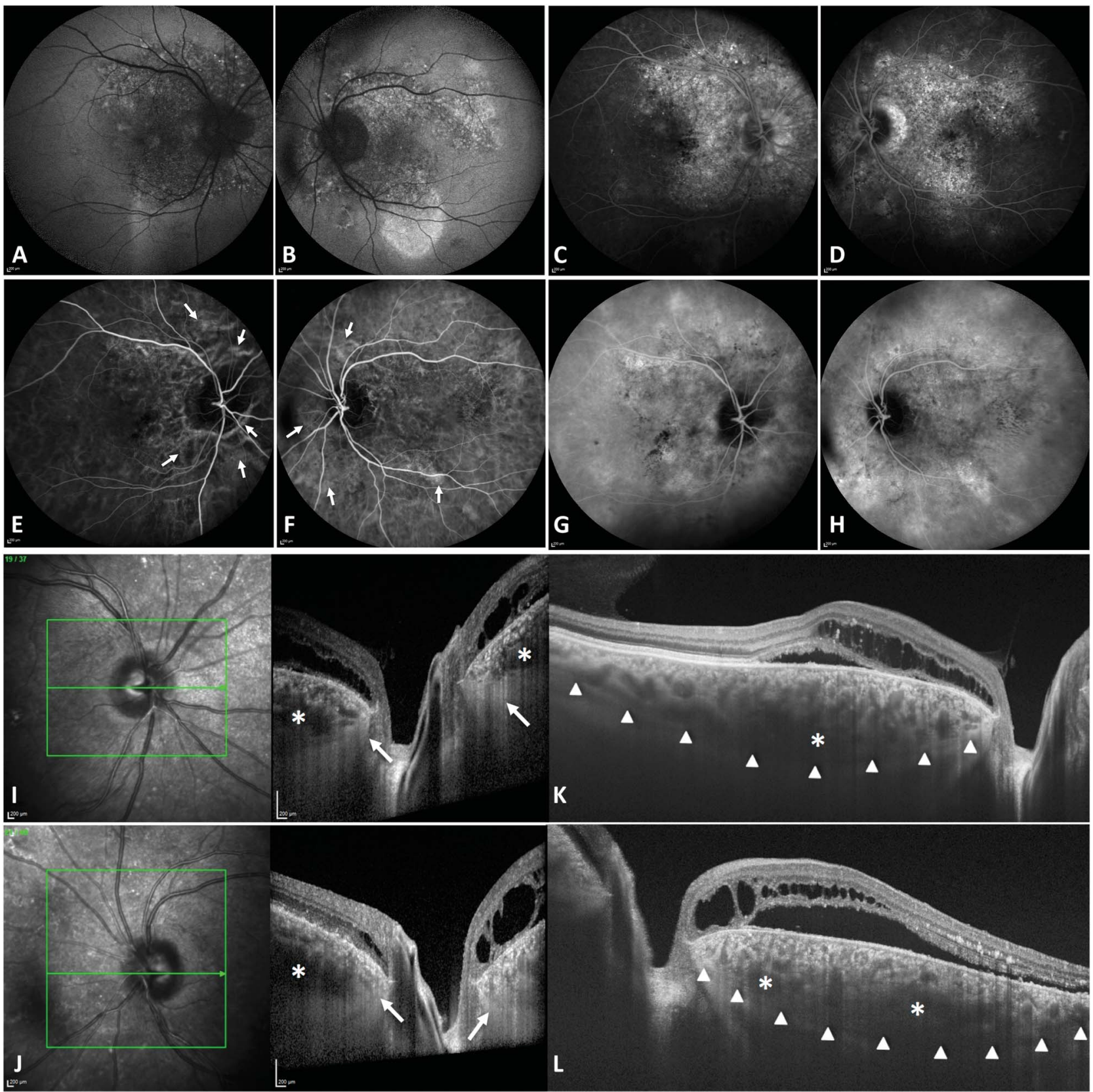

K
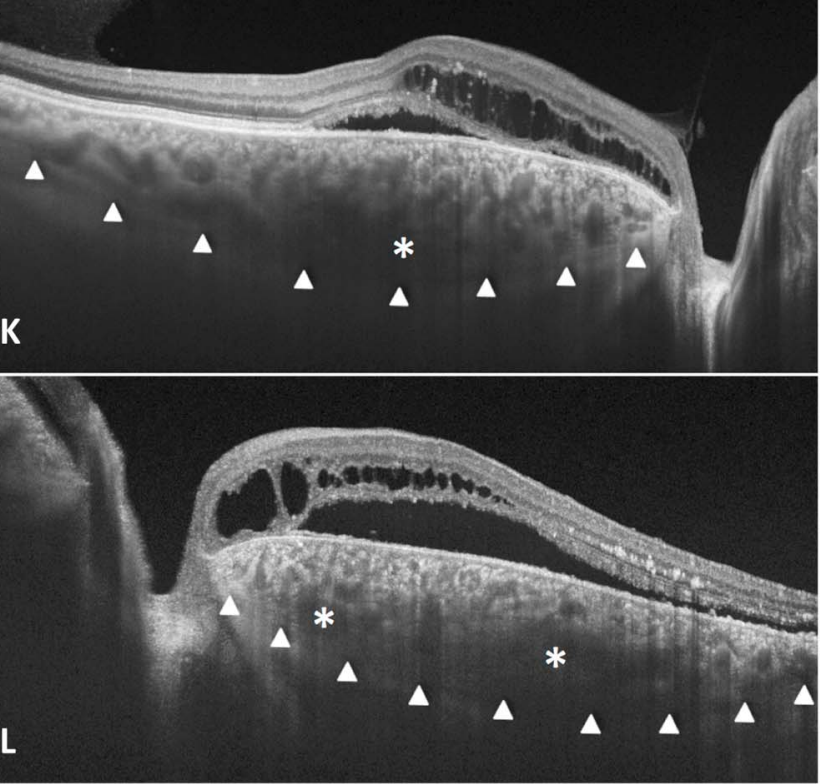

Fig. 2. Multimodal imaging of a 63-year-old man with PPS. A and B. Fundus autofluorescence illustrates hypoautofluorescent peripapillary atrophy (PPA) and large areas of peripapillary mottled autofluorescence in both eyes. Hyperautofluorescent patches are illustrated superotemporal in the left eye and inferior in both eyes and correspond to outer retinal atrophy similar to chronic CSC. C and D. Late-phase FA illustrates speckled hyperfluorescent window defects and staining surrounding the optic disk and intense staining of PPA in both eyes. There is no distinct leakage. $\mathbf{E}$ and $\mathbf{F}$. Early-phase ICGA illustrates peripapillary dilated large choroidal vessels (pachyvessels) (arrows). G and $\mathbf{H}$. Mid- to late-phase ICGA illustrates multifocal peripapillary choroidal hyperpermeability. I and J. OCT through the optic disks of the right (I) and left (J) eyes illustrates intraretinal fluid in both the nasal and temporal regions adjacent to the optic disks with associated choroidal hypertransmission (arrows) and atrophy of the RPE, ellipsoid zone, and ELM. Note the peripapillary pachyvessels (asterisks). $\mathbf{K}$ and $\mathbf{L}$. Swept-source OCT illustrates intraretinal fluid in the nasal macula and a thickened choroid (outlined by arrowheads) with pachyvessels (asterisks) more prominent in the nasal versus the temporal areas. Note the presence of subretinal fluid in both eyes.

Measurements from the main reader (N.P.) were used for the analysis, whereas those from the second reader (M.A.) were only used to calculate intergrader agreement.
Juxtapapillary retinal structures. For Group 1, retinal structures at the temporal disk margin included in the macula OCT scans were evaluated for the presence of intraretinal fluid, subretinal fluid and 

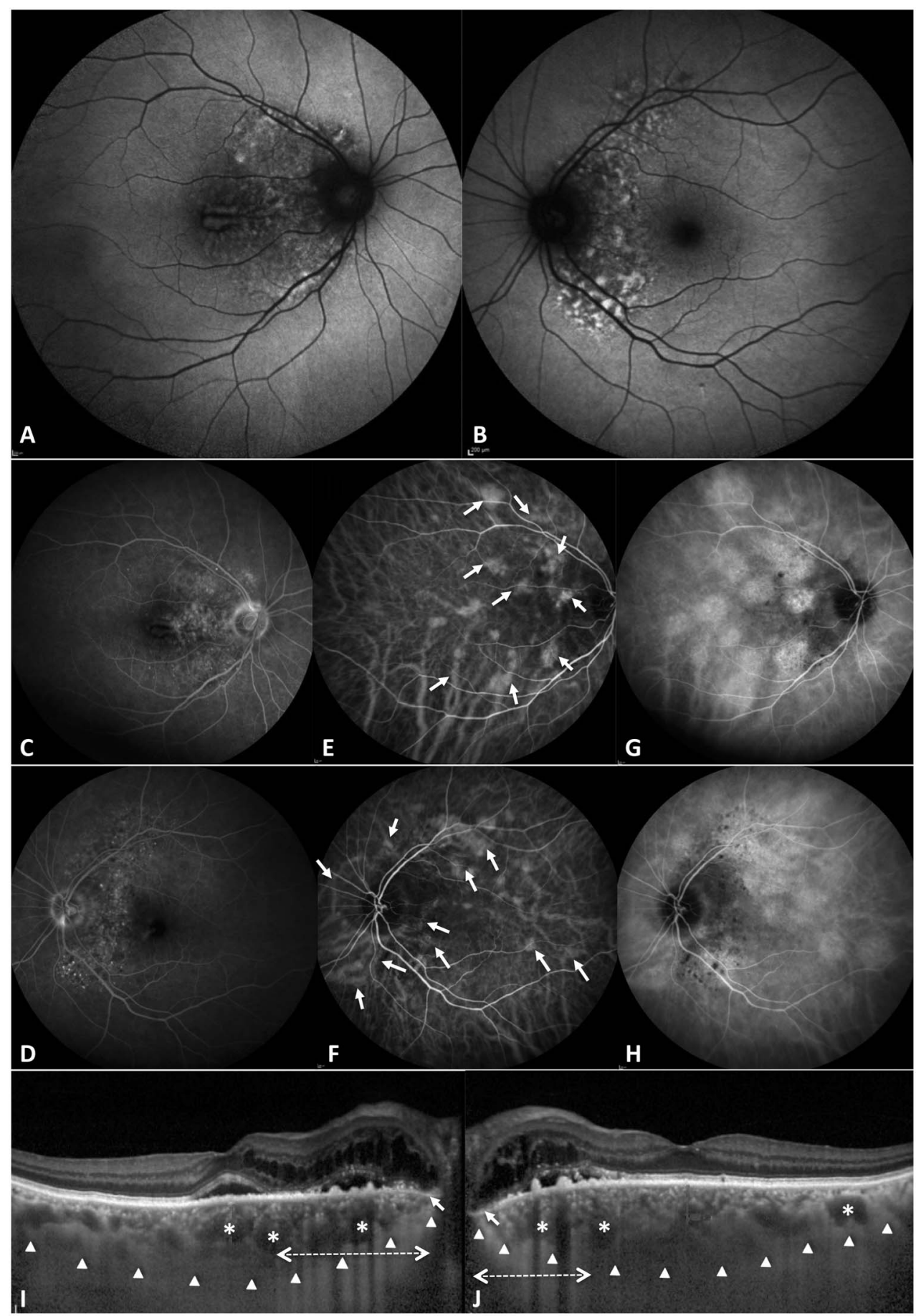

Fig. 3. Multimodal imaging of an 82-year-old man with PPS. A and $\mathbf{B}$. Fundus autofluorescence illustrates mottled autofluorescence of the RPE in the peripapillary region. $\mathbf{C}$ and $\mathbf{D}$. Late-phase FA illustrates peripapillary RPE window defects and mottled fluorescence without focal leakage in each eye. Note the peripapillary hyperfluorescent rings with mild late leakage in both eyes. $\mathbf{E}$ and $\mathbf{F}$. Early-phase ICGA illustrates dilated large choroidal vessels or pachyvessels (arrows) predominantly in the nasal macula and peripapillary region in both eyes. $\mathbf{G}$ and $\mathbf{H}$. Mid- to latephase ICGA illustrates multifocal choroidal hyperpermeability in areas corresponding to the pachyvessels. I and J. Enhanced depth imaging OCT illustrates intraretinal fluid with cysts in the nasal macula extending from the temporal optic disk margin and associated with focal RPE, ellipsoid zone, and ELM atrophy (solid arrows) in both eyes. Note the presence of RPE atrophy and the corresponding choroidal signal hypertransmission (dash arrows) in the peripapillary area in each eye. Note the thickened nasal macular choroid (outlined by arrowheads) associated with pachyvessels (asterisks) and thinning of the overlying inner choroid. The pachyvessels are more prominent in the nasal than temporal sides of the macula, similarly identified by ICGA. atrophy of RPE, ellipsoid zone, and external limiting membrane (ELM) with associated choroidal signal hypertransmission.

\section{Statistical Analysis}

Mean choroidal thickness at each position, mean age, and visual acuity among the three groups were compared. Snellen visual acuity was converted to logarithm of the minimum angle of resolution for statistical analysis. Ratios of nasal to temporal choroidal thickness were calculated and compared. The differences were tested using Welch test for analysis of variance with Games-Howell post hoc test for multiple comparisons. Chi-square or Fischer's exact test was used to test differences between proportions. $P$ value of 0.05 was set as a threshold of significance. 
Fig. 4. A 70-year-old man with PPS. A and B. Fundus autofluorescence illustrates hypoautofluorescent peripapillary atrophy and mottled autofluorescence at the temporal disk margin in both eyes with adjacent hyperautofluorescent patches. C and D. OCT through the optic nerve of the right $(\mathbf{C})$ and left (D) eyes illustrates intraretinal fluid extending from the temporal disk margin. $\mathbf{E}$ and F. Enhanced depth imaging OCT illustrates intraretinal cysts in the nasal macula extending from the disk margin with associated peripapillary atrophy with ellipsoid zone and ELM atrophy (solid arrows). Note the corresponding choroidal signal hypertransmission adjacent to the disk margins (dash arrows). The choroid is thicker with more dilated large choroidal vessels (asterisks) on the nasal side compared with the temporal side of the macula (outlined by arrowheads). $\mathbf{G}$ and $\mathbf{H}$. OCT B scans of the right $(\mathbf{G})$ and left (H) eyes at the level of the green lines in (A) and (B), respectively, illustrate choroidal folds.
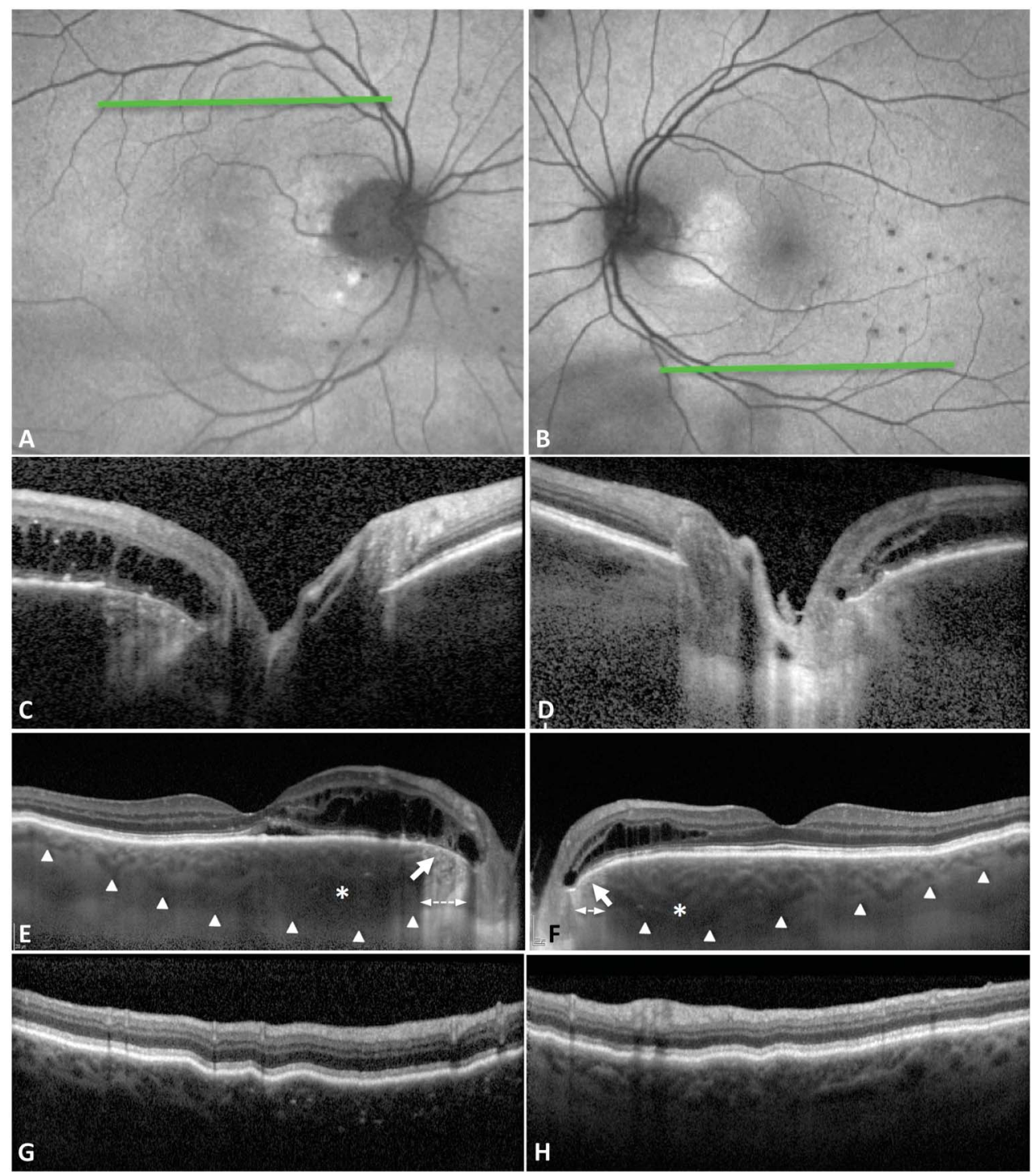

Intraclass correlation coefficients with $95 \%$ confidence intervals were calculated to evaluate intergrader agreement. Statistical analysis was performed using PASW Statistics for Windows, Version 18.0 (SPSS, Inc, Chicago, IL).

\section{Results}

A total of 31 eyes from 16 patients with peripapillary choroidal thickening associated with intraretinal and/or subretinal fluid extending from the temporal disk margin into the macula were identified (Group 1) comprising 13 men and 3 women with a mean age of 71 years (SD 7 years, range 58-86 years).

In Group 1, the choroid was thickened preferentially in the nasal macular region in contradistinction to those eyes with typical PDS and normal eyes as illustrated in Figures 1-7. Axial length (18 eyes) or manifest refraction (14 eyes) was available in 20 eyes and nearly all (12 of 14 eyes) were hyperopic with a mean $( \pm \mathrm{SD})$ spherical equivalence of +2.5 $( \pm 2.2)$ diopters. Axial lengths ranged from $20.00 \mathrm{~mm}$ to $24.59 \mathrm{~mm}$ (7 of 18 eyes (39\%) had axial lengths less than $23 \mathrm{~mm}$ ) with a mean $\pm \mathrm{SD}$ of $22.9 \pm 1.3 \mathrm{~mm}$. Choroidal folds were present in 24 of $31(77 \%)$ eyes often along the vascular arcades (Figures 4-6). The optic disk cup was small in most eyes with 18 of $31(58 \%)$ eyes with a cup less than 0.3 and 10 of $31(32 \%)$ with cupping of 0.1 or with a crowded disk (Figures 4 and 6). Chronic bilateral disk edema (Figure 7) was identified in 2 patients with normal neurological evaluations that included unremarkable magnetic resonance imaging of the brain and orbit and normal lumbar puncture.

Visual acuity in our patients with PPS was relatively good. Most patients $(65 \%, 20 / 31$ eyes) demonstrated 


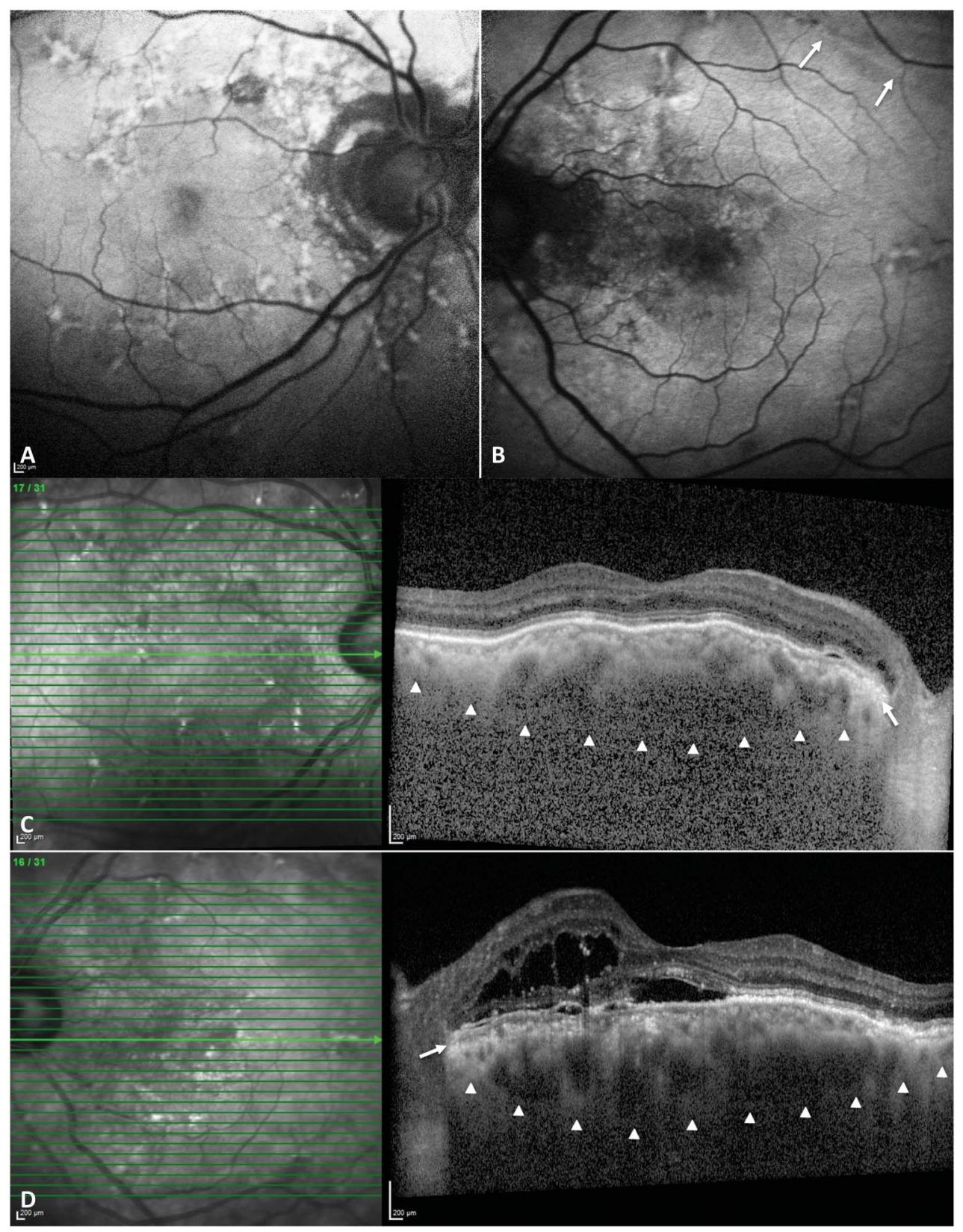

Fig. 5. Multimodal imaging of a 69-year-old man with PPS. A and $\mathbf{B}$. Fundus autofluorescence illustrates the predominant distribution of RPE abnormalities in the peripapillary region of both eyes. Alternate hyperautofluorescent and hypoautofluorescent bands of the choroidal folds are illustrated in the left eye (arrows). $\mathbf{C}$ and $\mathbf{D}$. Enhanced depth imaging OCT illustrates intraretinal cysts in the nasal macula that extend from the optic disk margin with associated atrophy of the RPE, ellipsoid zone, ELM, and the corresponding choroidal signal hypertransmission (arrows). Note also the presence of small pigment epithelial detachments in both eyes and subretinal fluid in the left eye. The choroid is thickened with more dilated large choroidal vessels predominantly in the nasal and central macular regions compared with the temporal area in each eye (outlined by arrowheads).

a visual acuity of 20/30 or better. Anatomical features associated with reduced visual acuity were intraretinal cysts approaching the foveal center, subfoveal fluid, and ellipsoid zone or RPE attenuation at the center of the fovea. The presence of optic disk edema without the above anatomical foveal abnormalities did not correlate with reduced visual acuity. Demographic and clinical data are summarized in Table 1.

Of the 31 Group 1 eyes, FA was available in 29 eyes, and FAF was available in 25 eyes. Peripapillary RPE alterations were identified in all 29 eyes with corresponding mottled autofluorescence or granular transmission hyperfluorescence on FA without focal areas of leakage. On FA, all eyes demonstrated late hyperfluorescent staining in a ring-like configuration immediately surrounding the optic disk with mild diffuse leakage in 14 eyes (Figure 8) and questionable mild leakage in another 10 eyes. Hyperautofluorescent patches in the posterior pole were found in 10 of 25 eyes with FAF assessment (Figures 2, 4, and 6). Gravitational tracks of pigmentary abnormalities on either FA or FAF were illustrated in 8 of 31 eyes (Figures 2 and 6). Indocyanine green angiography was available in 11 eyes and illustrated peripapillary dilated choroidal vessels with multifocal hyperpermeability in 9 eyes (Figures 2, 3, and 6). 


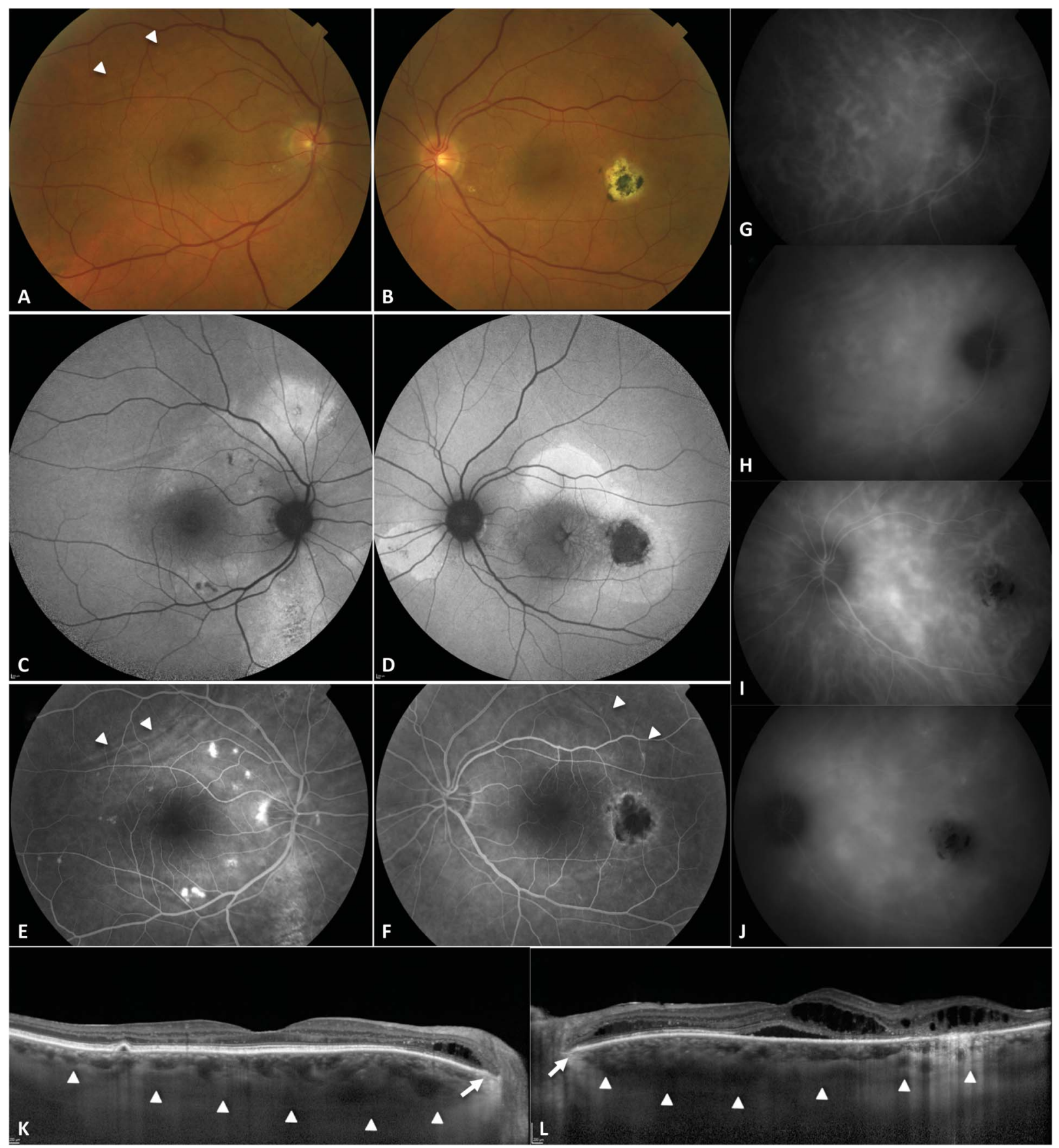

Fig. 6. Multimodal imaging of a 69-year-old man with PPS. A and B. Color fundus photographs illustrate juxtapapillary hard exudates in both eyes, mild pigmentary changes inferior to disk in the right eye, and a chorioretinal scar temporal to the macula in the left eye. Note the presence of choroidal folds (arrowheads) in the right eye and a small cup-to-disk ratio in both eyes. C and D. Fundus autofluorescence illustrates mild mottled autofluorescence temporal to the right disk and inferior to the left disk. A hyperautofluorescent patch superior to the disk and mottled autofluorescence inferior to the disk in a gravitational pattern are illustrated in the right eye. There are hyperautofluorescent patches inferonasal to the disk, superior to the fovea, and concentric to the hypoautofluorescent scar in the left eye. $\mathbf{E}$ and $\mathbf{F}$. FA illustrates multiple foci of window defects with late staining in the macula and peripapillary region, and multiple small pigment epithelial detachments in the right eye with a speckled hyperfluorescent gravitational tract extending from the disk inferiorly in the right eye. A hypofluorescent scar with late staining and variable staining in the peripapillary region are noted in the left eye. No significant fluorescein leakage is identified in either eye. Note the alternate hyperfluorescent and hypofluorescent bands of choroidal folds superotemporally in the right eye greater than the left eye (arrowheads). G-J. Indocyanine green angiography. Early ICGA (G and I) illustrates dilated large choroidal vessels in the peripapillary region and central macula with corresponding multifocal hyperpermeability in the midphase ICGA (H and $\mathbf{J}$ ) in both eyes. $\mathbf{K}$ and $\mathbf{L}$. Enhanced depth imaging OCT illustrates intraretinal fluid in the nasal macula extending from the disk margins with associated atrophy of the RPE, ellipsoid zone, ELM, and the corresponding choroidal signal hypertransmission (arrows) in both eyes. A temporal chorioretinal scar with surrounding intraretinal cysts and subfoveal fluid are also illustrated in the left eye. Note the thickened choroid (outlined by arrowheads) and dilated large choroidal vessels predominantly within the nasal region of each eye. 


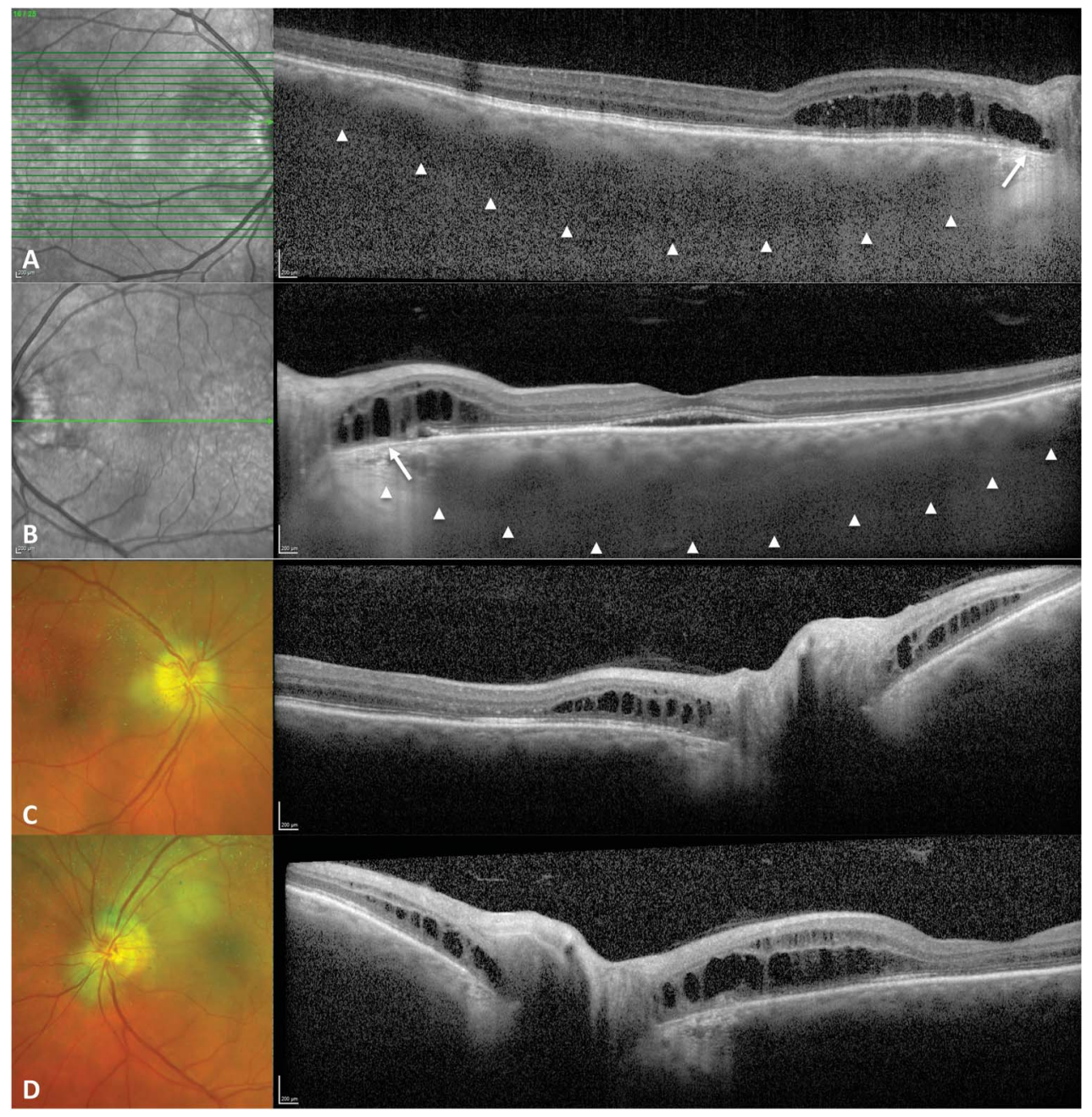

Fig. 7. Multimodal imaging of a 71-year-old man with PPS and chronic bilateral optic disk edema for over 1 year. The patient initially presented with bilateral enlarged blind spots and meticulous neurological workup including magnetic resonance imaging of the brain and orbits, lumbar puncture and cerebrospinal fluid analysis and infectious serology were all negative. $\mathbf{A}$ and B. Enhanced depth imaging OCT illustrates intraretinal fluid in the nasal macula extending from the optic disk margin in each eye and subretinal fluid in the left eye. Atrophy of the RPE, ellipsoid zone, and ELM is noted at the temporal disk margins and associated with corresponding choroidal signal hypertransmission (arrows). The choroid is thick with dilated large choroidal vessels more prominent in the nasal versus the temporal side in both eyes (outlined by arrowheads). C and D. OCT through the optic nerve head illustrates the presence of disk edema and intraretinal cysts surrounding the optic disk.

In addition to choroidal hyperpermeability, most eyes (19/27, 70\%) exhibited other features of the PDS including serous pigment epithelial detachment (13/ $31,42 \%$ ), FAF evidence of previous serous retinal detachment outside the peripapillary region $(15 / 25$, $60 \%$ ), and a gravitational track of RPE alteration (8/ 31, 26\%) (Figures 2 and 6).

Retinal structures at disk margins were studied by OCT. All eyes illustrated intraretinal fluid and cysts extending from the temporal disk margin with associated atropy of the RPE, ellipsoid zone, and ELM (Figures 2-7). These atrophic areas of the RPE and outer retina resulted in choroidal signal hypertransmission at the optic disk margin in all eyes. Varying amounts of subretinal fluid were present in 23 of 31 eyes. Optical coherence tomography B scans through the optic disks were available in 18 eyes, 11 of which demonstrated intraretinal fluid at the nasal disk margin (Figures 2 and 7).

To more accurately assess the significance of the choroidal thickness of the 31 eyes in Group 1 , comparative control groups were recruited to the study. Group 2 comprised 17 eyes from 11 patients and included eyes with typical CSC (13 eyes from 8 patients) or eyes with pachychoroid neovasculopathy (4 eyes from 3 patients). Group 3 comprised 19 normal eyes from 12 age-matched controls. The mean age was not statistically different between the 3 groups $(P=$ 0.117 ), but there were more women in Group 3 (Table 2). There was an excellent agreement in choroidal thickness measurements between the two readers with an intraclass correlation coefficient of 0.996 (95\% confidence interval, 0.995-0.997).

Analysis of choroidal thickness demonstrated significant differences among the three groups (Figure 9). The mean choroidal thickness in Group 1 (PPS) and Group 2 (typical PDS) was significantly greater than Group 3 (normal) at all 6 measured positions $(P<$ 0.01 ) except for position T3.0, where the difference between Group 1 and Group 3 was not statistically different $(P=0.06)$. Interestingly, Groups 1 and 2 illustrated different choroidal thickness profiles. Mean 
Table 1. Demographic and Clinical Data of Patients With PPS

\begin{tabular}{|c|c|c|c|c|c|c|c|c|c|}
\hline Patient & $\begin{array}{l}\text { Age, } \\
\text { years }\end{array}$ & Sex & Eye & VA & $\begin{array}{c}\text { Axial } \\
\text { Length, } \mathrm{mm}\end{array}$ & $\begin{array}{l}\text { Spherical } \\
\text { Equivalence }\end{array}$ & $\begin{array}{l}\text { Choroidal } \\
\text { Folds }\end{array}$ & $\begin{array}{c}\text { Cup-to-Disk } \\
\text { Ratio }\end{array}$ & Underlying Diseases \\
\hline \multirow[t]{2}{*}{1} & 65 & $\mathrm{~F}$ & OD & $20 / 50$ & 23.8 & +6.50 & Present & 0.4 & Monoclonal \\
\hline & & & OS & $20 / 30$ & 23.7 & +5.75 & Present & 0.4 & $\begin{array}{l}\text { gammopathy of } \\
\text { uncertain significance, } \\
\text { Raynaud disease, } \\
\text { Chiari I malformation }\end{array}$ \\
\hline \multirow[t]{2}{*}{2} & 86 & $\mathrm{~F}$ & OD & $20 / 30$ & 21.9 & NA & Present & 0.2 & Hypertension, \\
\hline & & & OS & $20 / 60$ & 21.8 & NA & Present & 0.3 & $\begin{array}{c}\text { hypercholesterol, sick } \\
\text { sinus syndrome, } \\
\text { breast cancer status } \\
\text { post mastectomy and } \\
\text { radiation }\end{array}$ \\
\hline \multirow[t]{2}{*}{3} & 75 & M & OD & $20 / 40$ & 23.7 & +2.00 & Present & 0.2 & Hypertension, sleep \\
\hline & & & OS & $20 / 25$ & 23.5 & +2.00 & Present & 0.3 & apnea \\
\hline \multirow[t]{2}{*}{4} & 71 & M & OD & $20 / 20$ & 23.1 & +3.75 & Present & 0.1 , edema & Hyperlipidemia, prostrate \\
\hline & & & OS & $20 / 40$ & 22.8 & +4.125 & Present & 0.1 , edema & $\begin{array}{l}\text { cancer (inactive), } \\
\text { transient ischemic } \\
\text { attack, anxiety }\end{array}$ \\
\hline \multirow[t]{2}{*}{5} & 69 & M & OD & $20 / 25$ & 23.6 & -0.75 & Present & 0.2 & Diabetes mellitus Type 2 \\
\hline & & & OS & $20 / 25$ & 23.8 & -0.75 & Present & 0.3 & \\
\hline \multirow[t]{2}{*}{6} & 70 & M & OD & $20 / 50$ & 22.6 & +2.00 & Present & 0.2 , edema & Multiple myeloma \\
\hline & & & OS & $20 / 25$ & 22.1 & +2.50 & Present & 0.3 , edema & \\
\hline \multirow[t]{2}{*}{7} & 79 & M & OD & $20 / 20$ & 24.6 & NA & Absent & 0.3 & None \\
\hline & & & OS & $20 / 30$ & 24.4 & NA & Absent & 0.3 & \\
\hline \multirow[t]{2}{*}{8} & 73 & $\mathrm{~F}$ & OD & $20 / 25$ & NA & NA & Present & 0.1 & Hypertension, melanoma \\
\hline & & & OS & $20 / 40$ & NA & NA & Present & 0.1 & $\begin{array}{l}\text { of the foot and leg, } \\
\text { Raynaud disease, } \\
\text { osteoarthritis, } \\
\text { osteopenia }\end{array}$ \\
\hline \multirow[t]{2}{*}{9} & 63 & M & OD & $20 / 40$ & 20.0 & NA & Present & 0.3 & Hypertension \\
\hline & & & OS & $20 / 120$ & 20.0 & NA & Present & 0.3 & \\
\hline \multirow[t]{2}{*}{10} & 67 & M & OD & $20 / 25$ & NA & +4.50 & Present & 0.4 & Kidney failure with renal \\
\hline & & & OS & $20 / 30$ & NA & NA & Present & 0.4 & $\begin{array}{c}\text { artery stenosis, } \\
\text { noninvasive bladder } \\
\text { tumors }\end{array}$ \\
\hline \multirow[t]{2}{*}{11} & 73 & M & OD & $20 / 20$ & NA & NA & Absent & 0.1 & End-stage renal disease \\
\hline & & & OS & $20 / 60$ & NA & NA & Absent & 0.1 & $\begin{array}{l}\text { status post kidney } \\
\text { transplantation, } \\
\text { coronary artery } \\
\text { disease, diabetes } \\
\text { mellitus, hypertension, } \\
\text { hyperlipidemia }\end{array}$ \\
\hline \multirow[t]{2}{*}{12} & 75 & M & OD & $20 / 25$ & NA & NA & Present & 0.1 & Diabetes mellitus, \\
\hline & & & OS & $20 / 30$ & NA & NA & Present & 0.1 & $\begin{array}{c}\text { hypertension, } \\
\text { hyperlipidemia, sleep } \\
\text { apnea, coronary artery } \\
\text { disease, anemia, } \\
\text { pancreatic cyst, } \\
\text { chronic renal } \\
\text { insufficiency }\end{array}$ \\
\hline \multirow[t]{2}{*}{13} & 70 & M & OD & $20 / 80$ & 23.8 & +1.25 & Present & 0.1 & Hypertension, \\
\hline & & & OS & $20 / 25$ & 23.9 & +0.50 & Present & 0.1 & hyperlipidemia \\
\hline \multirow[t]{2}{*}{14} & 82 & M & $\mathrm{OD}$ & $20 / 25$ & NA & NA & Present & 0.25 & Chronic kidney disease, \\
\hline & & & OS & $20 / 25$ & NA & NA & Present & 0.2 & $\begin{array}{c}\text { polymyalgia } \\
\text { rheumatica (on } \\
\text { prednisolone) }\end{array}$ \\
\hline \multirow[t]{2}{*}{15} & 65 & M & OD & $20 / 250$ & NA & NA & Absent & 0.2 & Diabetes mellitus Type 2 \\
\hline & & & OS & $20 / 30$ & NA & & Absent & 0.3 & \\
\hline 16 & 58 & M & OD & $20 / 25$ & NA & +2.00 & Absent & 0.2 & None \\
\hline
\end{tabular}

NA, not available. 
subfoveal choroidal thickness was not statistically different between these 2 groups; however, the mean choroidal thickness in the nasal macula in Group 1 was significantly greater than that of Group 2 at positions N1.5 and N3.0 $(P=0.038$ and 0.006 , respectively). The choroidal thickness of Group 1 then decreased sharply from the center position toward the temporal macula and was thinner than Group 2 temporally. In Group 1, the temporal macular choroid was even thinner than the nasal side. By contrast, the temporal macular choroid of Group 2 remained relatively thicker than the nasal side, demonstrating the same pattern as Group 3 (normal).

As the choroidal thickness was evidently thickened in the peripapillary region in Group 1, we performed an additional analysis of the ratio of nasal to temporal choroidal thickness. The ratios of choroidal thickness at N3.0 to T3.0 were $1.33 \pm$ $0.60,0.64 \pm 0.18$, and $0.49 \pm 0.17$ for Groups 1 , 2 , and 3, respectively. The ratios of choroidal thickness at $\mathrm{BMO} 250$ to $\mathrm{T} 3.0$ were $0.65 \pm 0.23,0.35 \pm$ 0.13 , and $0.31 \pm 0.14$ for Groups 1,2 , and 3, respectively. Furthermore, the ratios of the sum of choroidal thickness at N1.5 and N3.0 to the sum of that at $\mathrm{T} 1.5$ and $\mathrm{T} 3.0$ were $1.36 \pm 0.43,0.80 \pm 0.18$, and $0.65 \pm 0.20$ for Groups 1, 2, and 3, respectively. The differences between all these ratios of Group 1 versus Group 2 were all statistically significant $(P<$ $0.00001)$. Figure 10 demonstrates the distribution of eyes with various ratios of nasal to temporal choroidal thickness. Group 1 was considerably different from Groups 2 to 3. There were no eyes with an N3.0/T3.0 ratio greater than 1 in Groups 2 and 3, whereas 23/31 (74\%) of eyes in Group 1 had an N3.0/T3.0 ratio greater than $1\left(P<10^{-7}\right)$.

Data regarding treatment response were available for some study eyes. Three eyes treated with intravitreal anti-vascular endothelial growth factor therapy illustrated no response. One eye had resolution of macular fluid after verteporfin photodynamic therapy, whereas two other eyes showed no response. Two eyes treated with topical dorzolamide had resolution of macular fluid, whereas one eye showed minimal improvement. Oral acetazolamide in one patient was not associated with a reduction in macular fluid. One eye had spontaneous resolution of fluid with observation.

\section{Discussion}

We studied eyes with peripapillary choroidal thickening associated with intraretinal and/or subretinal fluid extending from the temporal disk margin into the macula. Our analysis demonstrated that the nasal macular choroid was significantly thicker than the temporal macular choroid in these eyes (Group 1), especially when compared with normal or typical PDS eyes. Most PPS eyes demonstrated choroidal folds (24/ $31,77 \%$ ), a relatively short axial length ( 7 of 18 eyes [39\%] with axial lengths less than $23 \mathrm{~mm}$ ) and a hyperopic refractive error $(12 / 14,86 \%)$. Many eyes $(11 / 18,61 \%)$ with available OCT B scans through the optic disk also illustrated intraretinal fluid on the nasal side of the nerve. The optic disk of these patients was typically crowded, and a small cup was identified in more than half of the cases with optic disk edema noted in four eyes of two cases. This unique clinical presentation prompted us to coin the term "peripapillary pachychoroid syndrome." The findings of PPS were bilateral in 15 of our 16 cases.

Our analysis illustrated that the pattern of choroidal thickness in our PPS eyes was significantly $(P<$ 0.0001 ) different than that of typical PDS entities such as CSC and pachychroid neovasculopathy and normal controls. Studies have illustrated that the macular choroidal thickness is normally greatest in the subfoveal position followed by the temporal and nasal positions. ${ }^{9,10}$ This was remarkably different from the pattern identified in PPS eyes, in which the nasal macular choroid was disproportionately thicker with associated pachyvessels compared with normal or typical PDS eyes and often thicker than the temporal macular choroid of the same eye (Figures 1, 9, and 10). Peripapillary choroidal thickening was associated with peripapillary RPE mottling on FAF or FA in all cases, but FA did not show focal leakage. Mild optic disk leakage was observed in 14/29 (48\%) eyes without evidence of ocular inflammation (Figure 8). Indocyanine green angiography demonstrated dilated peripapillary large choroidal vessels with multifocal choroidal hyperpermeability identified in the midphase ICGA.

The association of PPS with choroidal folds, short axial length, and hyperopia indicated similarities with UES, although there was no evidence of serous choroidal detachment in any of our cases. Recent case reports, ${ }^{6,11}$ with very similar findings to our cases of PPS, have applied the term "isolated posterior uveal effusion" to describe a syndrome characterized by a thick posterior choroid with serous macular detachment, nasal cystoid macular edema, choroidal folds, and short axial length without peripheral retinochoroidal detachment. However, we believe that our cases illustrated more in common with PDS rather than a form of UES for the following reasons: 1) Our patients often displayed serous RPE detachment and gravitational tracks of 


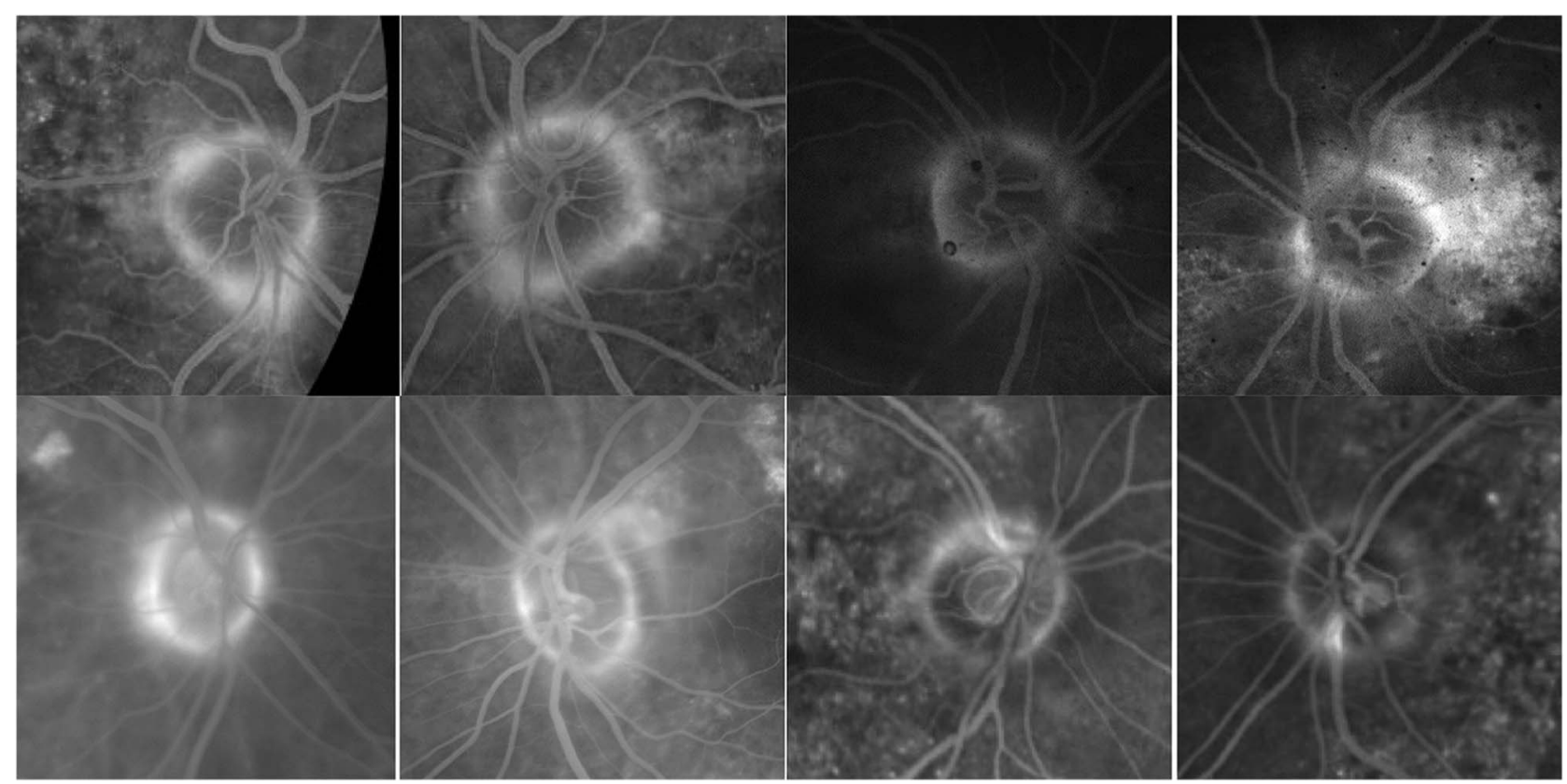

Fig. 8. Late FA illustrates peripapillary hyperfluorescent rings with mild late leakage in right and left eyes of 4 cases with PPS.

RPE alteration, which are commonly seen in PDS and have not been described in UES. In addition, pigmentary changes in a leopard-spot pattern characteristic of UES were not identified in our patients; 2) ICGA in our patients was very characteristic for PDS and included typical findings such as dilated large choroidal veins (pachyvessels) and choroidal hyperpermeability..$^{1,4,12,13}$ The pachyvessels were identified during dye transit and washed out in the late phase of the study. These pachyvessels corresponded to dilated Haller layer vessels visible on structural EDI-OCT and typically illustrated attenuation of the overlying Sattler layer and choriocapillaris. Indocyanine green angiography hyperpermeability was typically focal or multifocal, best seen during the midphase of the study, and often was observed around the washed out silhouettes of the pachyvessels. This hyperpermeability often faded in the late phase of the ICGA. By contrast, Uyama et $\mathrm{al}^{14}$ reported the ICGA pattern in UES as a diffusely granular hyperfluorescence in the very early phase, which increased with time and persisted until the late phase of the study as a diffuse intense choroidal hyperfluorescence; 3) The location of abnormal findings predominantly around the optic nerve and in the nasal macula was not consistent with UES and was much more typical of PDS/CSC. Hence, we prefer the term "peripapillary pachychoroid syndrome" for this entity. We have refined the clinical picture demonstrating that, unlike most other PDS disorders, the choroid in PPS is preferentially thickened in the nasal macula compared with the temporal macula. It is believed that the primary abnormality of the pachychoroid spectrum disorders relates to dilated choroidal vessels, ${ }^{3-5}$ whereas in UES, a reduced fluid and protein permeability of the sclera is the main mechanism. ${ }^{14,15}$ It is possible that reduced scleral permeability with older age ${ }^{16,17}$ may exacerbate peripapillary choroidal congestion in patients with PPS, giving rise to some overlapping findings with UES.

A recent large case series of patients with chorioretinal folds caused by various ocular disorders included eyes with mottled hyperfluorescence in the peripapillary and/or macular regions similar to our cases. ${ }^{18}$ However, EDI-OCT and choroidal imaging were not performed in these eyes. According to the findings of our study, PPS should also be considered in the differential diagnosis of chorioretinal folds.

Although certain findings in PPS eyes were suggestive of chronic CSC, another PDS entity, there were important distinguishing features. Overlapping findings included serous pigment epithelial detachment, gravitational tracks, evidence of serous retinal detachment outside the peripapillary region with FAF, and outer retinal atrophy in most $(19 / 27,70 \%)$ PPS eyes (Figures 2 and 6). Also, a male predilection (13/16, $81 \%$ ) and the ICGA findings of pachyvessels and hyperpermeability were similar to that of CSC. The most important feature distinguishing PPS from CSC was the preferential choroidal thickening in the peripapillary region as demonstrated in Figures 1, 9, and 10. The choroidal thickness profile was significantly different from typical CSC in that the nasal macular choroid was thickened and the thickness sharply decreased toward the temporal side. Other useful distinguishing features included the presence of choroidal 
Table 2. Demographic and Visual Acuity Data of the Three Groups of Subjects in This Study

\begin{tabular}{lcccc}
\hline & Group 1 (PPS) & Group 2 (PDS) & Group 3 (Normal) & $P$ \\
\hline Age, years & & & & \\
Mean \pm SD & $71.3 \pm 7.2$ & $63.7 \pm 9.7$ & $69.3 \pm 5.7$ & $61-79$ \\
Range & $58-86$ & $52-80$ & $4 / 8,33$ & 0.1170 \\
Sex, male/female, \%male & $13 / 3,81$ & $9 / 2,82$ & $0.0 \pm 0.1$ & 0.0004 \\
LogMAR VA, mean \pm SD & $0.2 \pm 0.2$ & $0.3 \pm 0.5$ & $20 / 20$ & \\
Mean Snellen VA & $20 / 32$ & $20 / 40$ & & \\
\hline
\end{tabular}

LogMAR, logarithm of the minimum angle of resolution.

folds, older age, and a small cup-to-disk ratio with mild disk leakage with late FA, not commonly present in CSC.

Multimodal imaging analysis in our study suggested that the primary abnormality of PPS resided in the peripapillary choroid where the characteristic PDS findings of ICGA hyperpermeability and pachyvessels were observed. Peripapillary choroidal congestion in PPS may lead to high hydrostatic pressure under the RPE causing RPE dysfunction and leakage of fluid into the subretinal space as has been postulated in the context of CSC. ${ }^{14,19}$ This hydrostatic pressure may be subject to a compartment-like syndrome (if choroidal outflow is disrupted) and may compress the optic nerve leading to complications including crowded disk, optic disk edema, and/or optic disk leakage.
Growing evidence of peripapillary pachychoroid exerting pressure stress on the optic nerve has recently emerged as a contributing factor for acquired lamina cribrosa defects ${ }^{20}$ and nonarteritic anterior ischemic optic neuropathy. ${ }^{21}$ The reason why the choroid is congested preferentially in the peripapillary region is unclear. Interestingly, choroidal venous drainage from the peripapillary choroidal region has been studied histologically. ${ }^{22}$ These veins penetrate the sclera around the optic nerve and enter the pia mater of the nerve and are called choroidopial veins. Whether there is an increased resistance of choroidal venous outflow through these choroidopial veins or the vortex veins in PPS requires further investigation.

The mechanism of intraretinal fluid extension from the disk margin in PPS is unclear. Fluorescein leakage

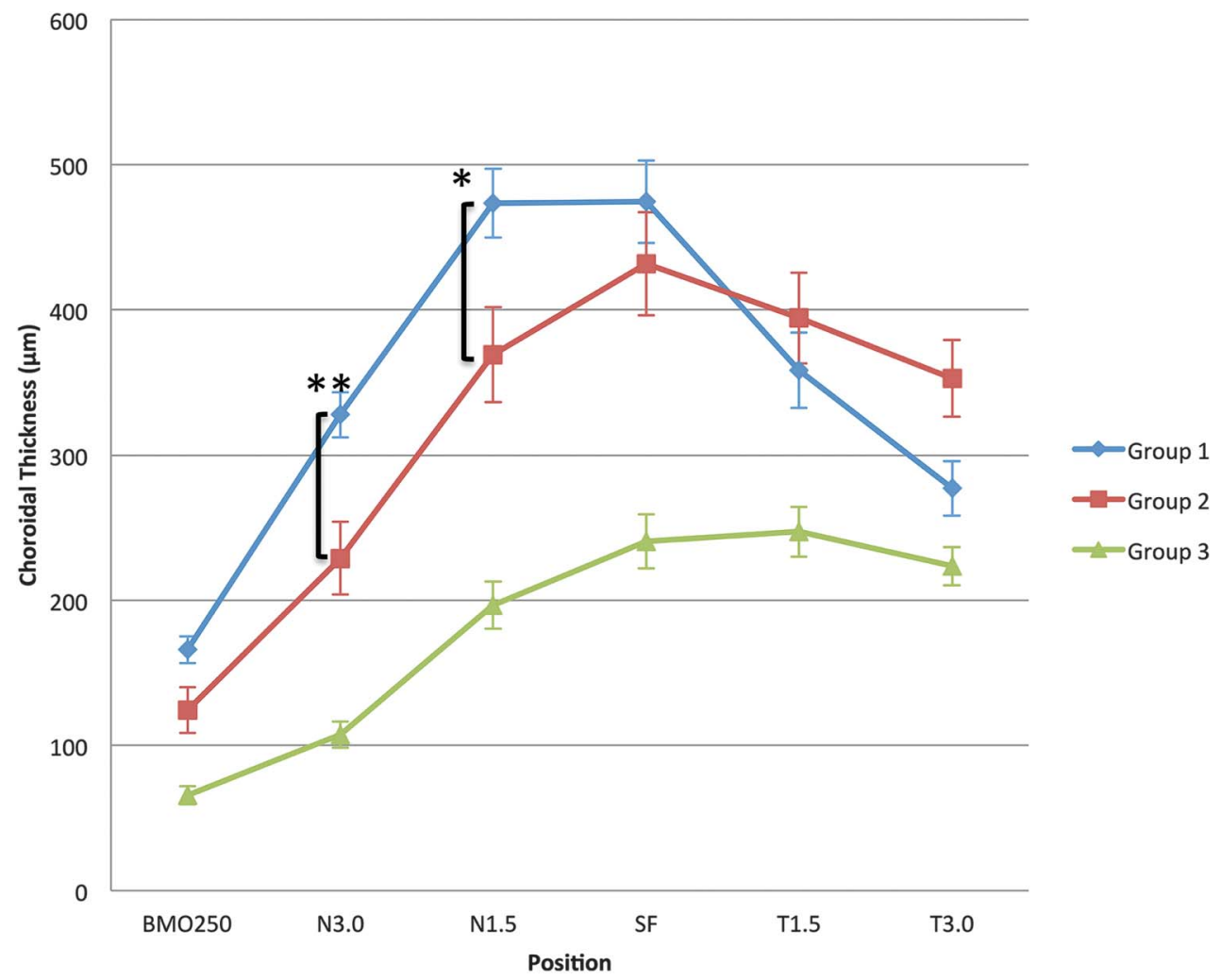

Fig. 9. This graph illustrates choroidal thickness at different positions in the macula of eyes with PPS. A distinctive choroidal thickness profile is noted. The nasal choroid is significantly thicker and sharply thins out toward the temporal side in the group of eyes with PPS versus the normal and the typical PDS groups. Group 1, PPS; Group 2, typical PDS (CSC or pachychoroid neovasculopathy); Group 3, age-matched normal eyes; BMO250, $250 \mu \mathrm{m}$ temporal to Bruch membrane origin; N3.0, 3,000 $\mu \mathrm{m}$ nasal to the foveal center; N1.5, 1,500 $\mu \mathrm{m}$ nasal to the foveal center; SF, subfoveal; T1.5, 1,500 $\mu \mathrm{m}$ temporal to the foveal center; and T3.0, 3,000 $\mu \mathrm{m}$ temporal to the foveal center. $P$ values were significant when the designated intervals were compared, $* P<$ 0.05 and $* * P<0.01$. 

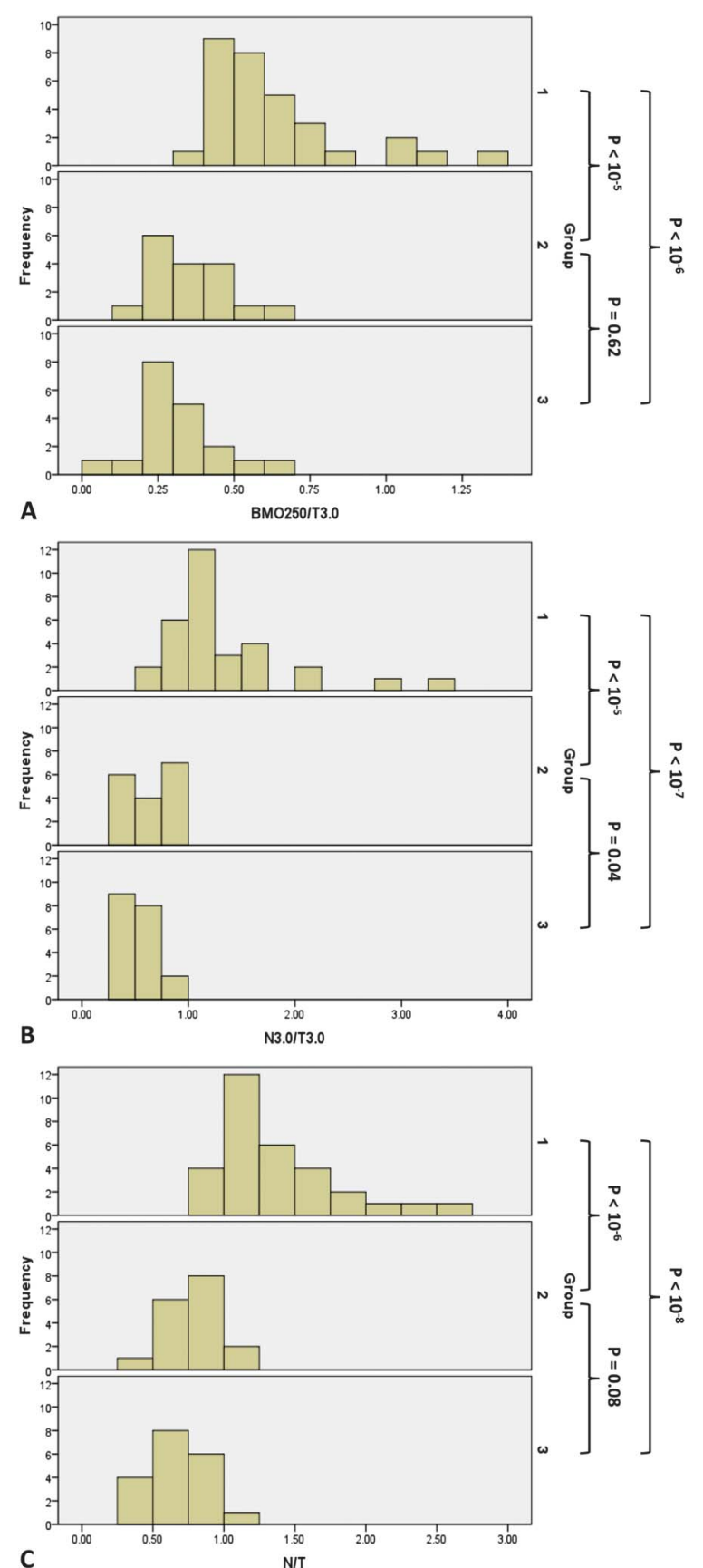

Fig. 10. Histograms illustrating distributions of different ratios of nasal to temporal choroidal thickness in each group. A. Ratio of choroidal thickness: BMO250 over T3.0. B. Ratio of choroidal thickness: N3.0 over T3.0. C. Ratio of nasal to temporal choroidal thickness $(\mathrm{N} / \mathrm{T}=$ $[\mathrm{N} 1.5+\mathrm{N} 3.0] /[\mathrm{T} 1.5+\mathrm{T} 3.0])$. The distribution of nasal to temporal choroidal thickness ratios illustrates a shift toward a value of 1 or greater for Group 1 (unlike Groups 2 and 3) indicating that the nasal choroid is thicker than the temporal choroid in contrast with Groups 2 and 3. $P$ values indicate comparisons of the mean ratios between each group. The differences between Group 1 versus Group 2 and Group 1 versus Group 3 were highly statistically significant. Group 1, PPS; Group 2, typical PDS (CSC or pachychoroid neovasculopathy); Group 3, age-matched normal eyes; BMO250, $250 \mu \mathrm{m}$ temporal to Bruch membrane origin; N3.0, 3,000 $\mu \mathrm{m}$ nasal to the foveal center; $\mathrm{N} 1.5$, $1,500 \mu \mathrm{m}$ nasal to the foveal center; T1.5, 1,500 $\mu \mathrm{m}$ temporal to the foveal center; T3.0, 3,000 $\mu \mathrm{m}$ temporal to the foveal center.

was minimal in PPS eyes and angiographic cystoid macular edema was not appreciated in these cases. It has been suggested that intraretinal cystic fluid may originate from the congested choroid through regions of peripapillary atrophy and associated atrophy of the RPE and ELM that normally serve as barriers for fluid to enter the retina. ${ }^{6}$ In this study, atrophy of the RPE and ELM and the corresponding choroidal signal hypertransmission at the temporal disk margins were detected by spectral domain OCT in all cases even in cases without frank peripapillary atrophy, and these juxtapapillary atrophic areas may facilitate fluid entry into the retina. Whether these atrophic areas are secondary to chronic hydrostatic stress to the optic disk margin from the congested peripapillary choroid or secondary to the presence of chronic subretinal fluid because of RPE dysfunction requires further longitudinal investigation. Alternatively, the intraretinal cysts without FA leakage may represent degenerative cavitation as has been described in the context of chronic $\mathrm{CSC}^{23-25}$ although the presence of SRF and large exudative-like retinal cysts in many cases makes cavitation unlikely.

A recent study by Lee et $\mathrm{al}^{20}$ described the presence of lamina cribrosa defects or disinsertions as a potential source of intraretinal fluid extending from the disk without leakage on FA in patients with pachychoroid disorders and patients with glaucomatous optic neuropathy. This study has proposed that a thickened choroid could stress the optic nerve head margin and lead to a lamina cribrosa defect or disinsertion and subsequent intraretinal fluid. Our study was retrospective and did not use an EDI-OCT raster protocol with sufficient density to detect such defects. Of note, the pachychoroid subjects in Lee's study seemed to be different from ours because Lee's study excluded eyes with peripapillary intraretinal fluid associated with RPE atrophy (peripapillary fluid and RPE atrophy were found in all cases in our study), and only two of eight eyes illustrated late staining of the optic nerve border (as opposed to all eyes in our study). Also, it was not clear in their study whether the macular choroid was preferentially thickened nasally as in PPS. Furthermore, the lamina cribrosa defects were not detected in about half of their pachychoroid patients. This indicates that there may be more than one mechanism of intraretinal fluid accumulation.

The association of a crowded disk appearance or optic disk edema with PPS is interesting. Sarraf and Schwartz, ${ }^{26}$ before the OCT era, described three cases of bilateral choroidal folds and short axial length associated with crowded disk and FA leakage in one eye and optic atrophy in the fellow eye. They excluded neurological disorders including idiopathic intracranial hypertension and proposed that idiopathic acquired 
hyperopia and shrinkage of the scleral canal could lead to a crowded disk syndrome and the development of nonarteritic anterior ischemic optic neuropathy. Some of our cases shared similar features of small optic cups, optic disk leakage, short axial lengths, and choroidal folds. These findings were similar to the crowded disk syndrome cases described by Sarraf and Schwartz, ${ }^{26}$ and it is possible that these cases would have demonstrated PPS with current multimodal imaging capabilities. As mentioned above, a finding of peripapillary pachychoroid has recently been implicated in nonarteritic anterior ischemic optic neuropathy. ${ }^{21}$ Whether patients with PPS have a higher incidence of nonarteritic anterior ischemic optic neuropathy requires further investigation, however.

It should be acknowledged that some eyes in our study could be classified with more than one PDS disorder (hence the term "spectrum"). Peripapillary pachychoroid syndrome is best defined by the presence of characteristic peripapillary abnormalities. In some eyes, this may be the primary pathology, but in others, these findings may be more of an incidental finding detected during an evaluation of some other disorders of the PDS such as CSC or polypoidal choroidal vasculopathy. In this study, eyes with Type 1 neovascularization were excluded from Group 1 analysis. However, PPS may be associated with Type 1 neovascularization with or without polyps.

Limitations of this analysis included the retrospective nature of the study, incomplete data collection and incomplete multimodal imaging in some cases, and the relatively small sample size. Choroidal analysis was compared with control eyes using a single-line EDIOCT scan through the foveal center. Future prospective and longitudinal studies with comparative volumetric OCT scans would be valuable to corroborate our findings.

In summary, we have described a new subgroup in the PDS referred to as PPS and characterized by a relatively thickened nasal macular choroid (vs. the temporal choroid) with associated intraretinal and subretinal fluids in the nasal macular region extending from the disk margin. Intraretinal and subretinal fluids can also be identified nasal to the optic disk. Focal RPE and ELM atrophy at the disk margin and corresponding choroidal hypertransmission were identified in all eyes and may indicate the site of fluid entry into the retina. Fundus autofluorescence and FA illustrated peripapillary mottling of the RPE with late staining but minimal or no leakage. Indocyanine green angiography demonstrated pachyvessels and multifocal hyperpermeability in the peripapillary region. The optic nerve head was usually crowded with mild late leakage with FA and even edematous in certain cases.
Patients with PPS were mostly men but typically older than those with CSC. Choroidal folds, a relatively short axial length and hyperopia were common associations. Peripapillary choroidal congestion with a compartment-like effect on the peripapillary region was proposed as an etiologic mechanism. Although PPS may be considered in the spectrum of pachychoroid disorders such as CSC, the aggregate of presenting features are sufficiently characteristic as to be considered a unique syndrome. It is important to recognize PPS and distinguish this new clinical entity from similar disorders such as posterior uveitis and neuro-ophthalmologic conditions that can share overlapping features, namely disk leakage and edema.

Key words: central serous chorioretinopathy, choroidal folds, choroidal thickness, optic disk edema, pachychoroid disease spectrum.

\section{References}

1. Warrow DJ, Hoang QV, Freund KB. Pachychoroid pigment epitheliopathy. Retina 2013;33:1659-1672.

2. Chung H, Byeon SH, Freund KB. Focal choroidal excavation and its association with pachychoroid spectrum disorders: a review of the literature and multimodal imaging findings. Retina 2017;37:199-221.

3. Dansingani KK, Balaratnasingam C, Naysan J, Freund KB. En face imaging of pachychoroid spectrum disorders with sweptsource optical coherence tomography. Retina 2016;36:499-516.

4. Pang CE, Freund KB. Pachychoroid neovasculopathy. Retina 2015;35:1-9.

5. Balaratnasingam C, Lee WK, Koizumi H, et al. Polypoidal choroidal vasculopathy: a distinct disease or manifestation of many? Retina 2016;36:1-8.

6. Pautler SE, Browning DJ. Isolated posterior uveal effusion: expanding the spectrum of the uveal effusion syndrome. Clin Ophthalmol 2015;9:43-49.

7. Yiu G, Pecen P, Sarin N, et al. Characterization of the choroidscleral junction and suprachoroidal layer in healthy individuals on enhanced-depth imaging optical coherence tomography. JAMA Ophthalmol 2014;132:174-181.

8. Schneider CA, Rasband WS, Eliceiri KW. NIH Image to ImageJ: 25 years of image analysis. Nat Methods 2012;9:671-675.

9. Shin JW, Shin YU, Lee BR. Choroidal thickness and volume mapping by a six radial scan protocol on spectral-domain optical coherence tomography. Ophthalmology 2012;119:1017-1023.

10. Margolis R, Spaide RF. A pilot study of enhanced depth imaging optical coherence tomography of the choroid in normal eyes. Am J Ophthalmol 2009;147:811-815.

11. Liu Q, Hemarat K, Kayser DL, Stewart JM. A case of posterior uveal effusion syndrome masquerading as uveitis. Retin Cases Brief Rep 2016;1:S124-S127.

12. Dansingani KK, Balaratnasingam C, Klufas MA, et al. Optical coherence tomography angiography of shallow irregular pigment epithelial detachments in pachychoroid spectrum disease. Am J Ophthalmol 2015;160:1243-1254.e2.

13. Ersoz MG, Arf S, Hocaoglu M, et al. Indocyanine green angiography of pachychoroid pigment epitheliopathy. Retina 2017.

14. Uyama M, Takahashi K, Kozaki J, et al. Uveal effusion syndrome: clinical features, surgical treatment, histologic exami- 
nation of the sclera, and pathophysiology. Ophthalmology 2000;107:441-449.

15. Elagouz M, Stanescu-Segall D, Jackson TL. Uveal effusion syndrome. Surv Ophthalmol 2010;55:134-145.

16. Jackson TL, Hussain A, Morley AM, et al. Scleral hydraulic conductivity and macromolecular diffusion in patients with uveal effusion syndrome. Invest Ophthalmol Vis Sci 2008; 49:5033-5040.

17. Stewart JM, Schultz DS, Lee OT, Trinidad ML. Exogenous collagen cross-linking reduces scleral permeability: modeling the effects of age-related cross-link accumulation. Invest Ophthalmol Vis Sci 2009;50:352-357.

18. Olsen TW, Palejwala NV, Lee LB, et al. Chorioretinal folds: associated disorders and a related maculopathy. Am J Ophthalmol 2014;157:1038-1047.

19. Guyer DR, Yannuzzi LA, Slakter JS, et al. Digital indocyanine green videoangiography of central serous chorioretinopathy. Arch Ophthalmol 1994;112:1057-1062.

20. Lee JH, Park HY, Baek J, Lee WK. Alterations of the lamina cribrosa are associated with peripapillary retinoschisis in glau- coma and pachychoroid spectrum disease. Ophthalmology 2016;123:2066-2076.

21. Nagia L, Huisingh C, Johnstone J, et al. Peripapillary pachychoroid in nonarteritic anterior ischemic optic neuropathy. Invest Ophthalmol Vis Sci 2016;57:4679-4685.

22. Ruskell GL. Peripapillary venous drainage from the choroid: a variable feature in human eyes. $\mathrm{Br} \mathrm{J}$ Ophthalmol 1997;81:76-79.

23. Iida T, Yannuzzi LA, Spaide RF et al. Cystoid macular degeneration in chronic central serous chorioretinopathy. Retina 2003;23:1-7; quiz 137-138.

24. Piccolino FC, De La Longrais RR, Manea M, Cicinelli S. Posterior cystoid retinal degeneration in central serous chorioretinopathy. Retina 2008;28:1008-1012.

25. Piccolino FC, De La Longrais RR, Manea M, et al. Risk factors for posterior cystoid retinal degeneration in central serous chorioretinopathy. Retina 2008;28:1146-1150.

26. Sarraf D, Schwartz SD. Bilateral choroidal folds and optic neuropathy: a variant of the crowded disk syndrome? Ophthalmology 2003;110:1047-1052. 